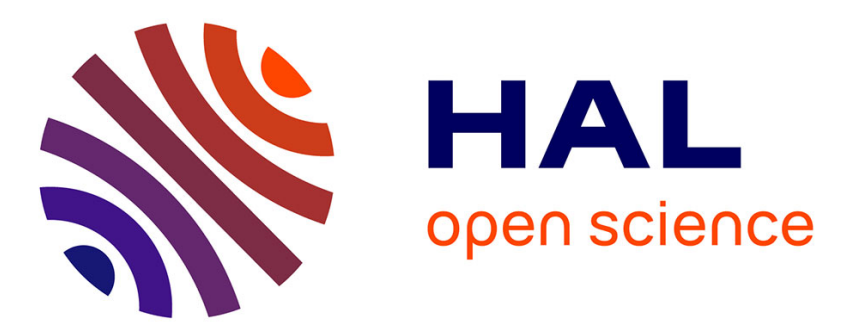

\title{
Augmented 3D Catheter Navigation using Constrained Shape from Template
}

Raffaella Trivisonne, Erwan Kerrien, Stéphane Cotin

\section{To cite this version:}

Raffaella Trivisonne, Erwan Kerrien, Stéphane Cotin. Augmented 3D Catheter Navigation using Constrained Shape from Template. Hamlyn Symposium, Jun 2017, London, United Kingdom. hal01545693

\section{HAL Id: hal-01545693 \\ https://inria.hal.science/hal-01545693}

Submitted on 23 Jun 2017

HAL is a multi-disciplinary open access archive for the deposit and dissemination of scientific research documents, whether they are published or not. The documents may come from teaching and research institutions in France or abroad, or from public or private research centers.
L'archive ouverte pluridisciplinaire HAL, est destinée au dépôt et à la diffusion de documents scientifiques de niveau recherche, publiés ou non, émanant des établissements d'enseignement et de recherche français ou étrangers, des laboratoires publics ou privés. 


\title{
Augmented 3D Catheter Navigation using Constrained Shape from Template
}

\author{
Raffaella Trivisonne ${ }^{1}$, Erwan Kerrien ${ }^{1}$, Stéphane Cotin ${ }^{1}$ \\ ${ }^{1}$ Inria, France \\ raffaella.trivisonne@inria.fr
}

\section{INTRODUCTION}

Endovascular surgery is a medical specialty of minimally invasive procedures, which relies on the use of catheters, guide-wires and other endovascular devices, to reach and treat a variety of pathologies. During the intervention, fluoroscopic images provide the clinician with live feedback on the anatomy of the patient, and the position and shape of the catheter. In conventional 2D fluoroscopic guidance, the complexity of the procedure, the radiation exposure and the loss of depth perception in X-ray images, are reasons why improved means of visualization have been investigated Commercial systems are already available [5], fusing live X-ray images with pre-acquired 3D MRI or CT images. This can be further improved by reproducing the 3D navigation of the catheter, which would otherwise remain 2D. Several approaches have been proposed, based on computer vision, numerical simulations, or the use of physical sensors ([8], [1], [7]). In [6] the authors present a method to retrieve the 3D position of the catheter combining the 2D fluoroscopic view with pre-operative models of the vasculature. In this context of augmented endovascular procedures, we propose a method to retrieve the $3 \mathrm{D}$ navigation of the catheter from a single view scenario, without the use of external sensor. Using a combination of visual features extracted from the fluoroscopic image, and the registered pre-operative $3 \mathrm{D}$ vessel surface, we drive a constraint-based numerical simulation, which allows us to retrieve the 3D catheter shape from fluoroscopic images. This will allow clinicians to virtually explore different angles of view without moving the imaging equipment.

\section{MATERIALS AND METHODS}

To reconstruct the 3D shape of a flexible device, we perform a real-time physics-based simulation of the flexible tool constrained by information extracted from the live fluoroscopic data and boundary conditions arising from the pre-operative vascular surface. The mechanical consistency provided by the physics-based model of the flexible tool avoids ambiguous situations and provides a complete and accurate $3 \mathrm{D}$ visualization of the catheter. The model of the surgical tool is based on Timosenko beam theory, solved using an efficient finite element method and a co-rotational approach [4]; it is represented as a series of serially-linked beam elements, where each node has 6 degrees of freedom [2] and the mechanical parameters are coherent with the reality. Currently image-features are easily extracted and tracked using OpenCV; we used a catheter with radiopaque tags every $1 \mathrm{~cm}$ in order to have a higher gradient variation. We impose that the projection of each 3D node should coincide with its corresponding point onto the image; to do that, we allow each 3D nodes to slide along the line of sight, defined between its corresponding 2D point detected in the image and the optical center of the camera. Nevertheless, 2D image features alone are not sufficient to retrieve the 3D shape of the device. Assuming that the position of the point through which the catheter is inserted, is known in 3D (for instance, obtained thanks to makers located on the catheter sheath), we define a further geometrical constraint enforcing the catheter to slide along a direction which reproduces the orientation of the insertion valve. A set of unilateral constraints are applied along the shaft of catheter to prevent crossing the surface of the 3D anatomy. Each of these constraints applies 1 dof unilateral contact force along the normal of the triangulated vessel surface, in order to enforce Signorini conditions i.e. A contact force is applied if and only if a (set of) point(s) of the device model move(s) outside of vessel geometry (see [9] for instance). As all of these are linear constraints (i.e. sliding constraints on a line), they allow to solve the problem with a Lagrangian approach for FE models [4] where the global equation is expressed with a static formulation.

$$
\left(\begin{array}{ll}
\mathbf{K} & \mathbf{J}^{\mathrm{T}} \\
\mathbf{J} & \boldsymbol{\alpha} \mathrm{I}
\end{array}\right)\left(\begin{array}{c}
\Delta \mathbf{X} \\
\boldsymbol{\lambda}
\end{array}\right)=\left(\begin{array}{l}
\mathbf{0} \\
\mathbf{\delta}
\end{array}\right)
$$

Where for each iteration, $\mathbf{K}$ is the stiffness matrix of the $\mathrm{FE}$ model, $\Delta \mathbf{X}$ is the equilibrium state of the $3 \mathrm{D}$ positions of the catheter, $\boldsymbol{\delta}$ is the violation of the constraints and $\mathbf{J}$ is the Jacobian of the constraints.

\section{RESULTS}

We performed catheter insertion on a rigid phantom. Two data-set have been acquired through a 3D capable angiography $\mathrm{C}$-arm at low and high fluoroscopy frequency, performing some $3 \mathrm{D}$ acquisitions $(\mathrm{CBCT})$ to retrieve the real 3D position of the catheter and validate our simulation; 3D and 2D images are registered through calibration [3]. Beside the catheter navigation under 2D fluoroscopy and 3D scans to validate the reconstructed shape at times, we acquired some fluoroscopic images while shifting the table downwards, orthogonally to the direction of insertion, to simulate movement deriving from patient's breathing motion or machinery adjustment, showing the robustness of our method to important motion. We exploited the temporal 
coherence of the detected markers to bind the 2D markers with the corresponding $3 \mathrm{D}$ nodes of the catheter. To validate our registration, we computed the Euclidean distance between the simulated catheter and the real one, previously retrieved from segmentation. For the first data-set, we had an average error of $2.7 \pm 0.7 \mathrm{~mm}$, corresponding to $11 \%$ of vessel diameter; for the data-set at $7.5 \mathrm{pps}$, the higher acquisition rate produced more noisy and blurred images, entailing a higher error in the registration process $(4.8 \pm 0.8 \mathrm{~mm})$. Within this second data set (Fig.1), we wanted to test as well the robustness of our method to potential table movement or breathing motion of the patient, i.e. any potential movement in a direction different from the direction of insertion. For that, we shifted the table $50 \mathrm{~mm}$ downwards, under the assumption that the camera stays fixed. In this second phase, the registration error uniformly increases during the downwards shift $(8 \pm 0.6 \mathrm{~mm}$ average deviation) due to the reprojection error of the calibration matrix that is not uniform along the image. The surface's constraint was not active.

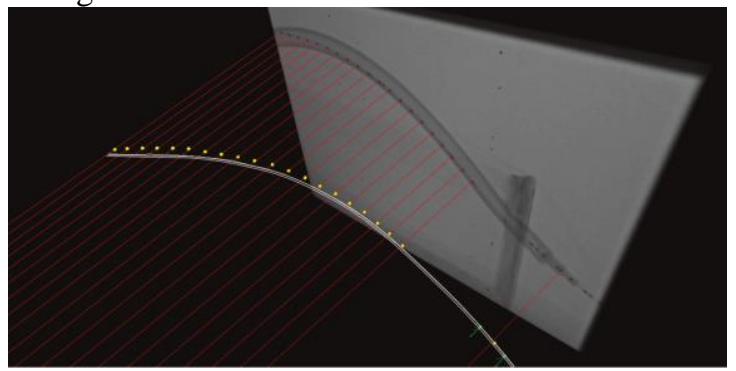

Fig. $13 \mathrm{D}$ catheter reconstruction for Real Dataset at the end of the insertion. Red lines represent the line of sight of projective constraints; yellow points represent the real catheter, obtained from segmentation of 3D medical images.

In any case, this kind of error can be easily compensated since the movement of the table can be known from the set-up parameters. Moreover, typical breathing motion of a patient reaches an amplitude of at most $2 \mathrm{~cm}$, i.e. inferior to the displacement imposed in this test. In order to evaluate our registration within a less trivial anatomy, we created a synthetic data-set simulating a catheter insertion. We recorded the images of the insertion, as under fluoroscopic guidance, and we used it as ground truth for the following registration. An average error of $0.8 \pm 0.07 \mathrm{~mm}$, corresponding to the $4 \%$ of the total diameter of the vessel, is registered (Fig.2). Detection errors and calibration errors, are compensated through empirical parameters $\alpha$ applied on the constraints.

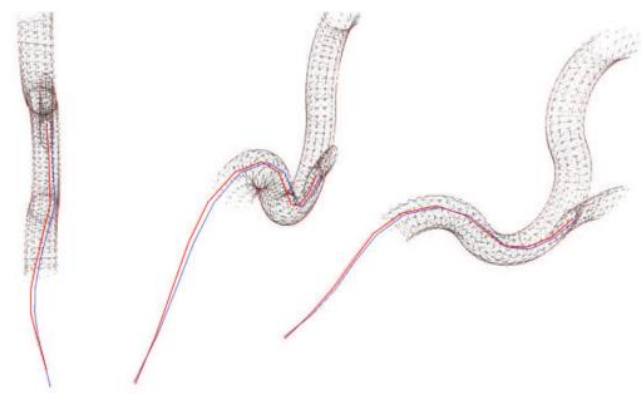

Fig. 2 Different views of 3D catheter reconstruction for Synthetic dataset; blue and red catheter are respectively the real and the simulated one.

\section{DISCUSSION}

In this work, we presented a single view reconstruction method to retrieve the 3D navigation of the catheter. Dealing with a rigid registration, this could be potentially applicable to neuro interventional radiology procedures, where brain's movement is limited. In order to progress towards different clinical application, we will investigate other detection methods to deal with guidewires or untagged catheters, and automatic calibration to deal with deformations and breathing motions. Eventually, we will exploit this simulation to reduce the acquisition frequency, where the information between two images will be provided by the simulation itself.

\section{REFERENCES}

[1] Condino, S., Ferrari, V., Freschi, C., Alberti, A., Berchiolli, R., Mosca, F., Ferrari, M.: Electromagnetic navigation platform for endovascular surgery: how to develop sensorized catheters and guidewires. The international journal of medical robotics + computer assisted surgery : MRCAS 8(3), 300-10 (2012).

[2] Cotin, S., Duriez, C., Lenoir, J., Neumann, P., Dawson, S.: New approaches to catheter navigation for interventional radiology simulation. MICCAI $8(\mathrm{Pt} 2)$, 534-42 (2005).

[3] Gorges, S., Kerrien, E., Berger, M.O., Trousset, Y., Pescatore, J., Anxionnat, R., Picard, L., Bracard, S.: 3D Augmented Fluoroscopy in Interventional Neuroradiology: Precision Assessment and First Evaluation on Clinical Cases (2006).

[4] Felippa, C.A., Haugen, B.: A unified formulation of small-strain corotational finite elements: I. Theory (2005).

[5] VesselNavigator by Philips

[6] Groher, M., Bender, F., Khamene, A., Wein, W., Heibel, T.H., Navab, N.: 3D Guide Wire Navigation from Single Plane Fluoroscopic Images in Abdominal Catheterizations. pp. 356-360.

[7] Pujol, S., Pecher, M., Magne, J.L., Cinquin, P.: A virtual reality based navigation system for endovascular surgery. Studies in health technology and informatics 98, 310-2 (2004).

[8] Schwein, A., Kramer, B., Chinna Durai, P., Walker, S., O’Malley, M., Lumsden, A., Bismuth, J.: Flexible Robotics With Electromagnetic Tracking Improve Safety and Efficiency During In Vitro Endovascular Navigation. Journal of Vascular Surgery 63(1), 285-286 (2016).

[9] Courtecuisse, H., Allard, J., Kerfriden, P., Bordas, S.P.A., Cotin, S., Duriez, C.:Real Time simulation of contact and cutting of heterogeneous soft-tissue. Med. Image Anal. 18(2) (2014) 394-410. 